\title{
Dark Clouds or Silver Linings? Knightian Uncertainty and Climate Change
}

\author{
$\mathrm{Yu}-\mathrm{Fu}$ Chen \\ Economic Studies \\ School of Business \\ University of Dundee \\ y.f.chen@dundee.ac.uk
}

\author{
Michael Funke \\ Department of Economics \\ Hamburg University \\ and CESifo, Munich \\ funke@econ.uni-hamburg.de
}

\author{
Nicole Glanemann \\ International Max Planck Research School \\ on Earth System Modelling (IMPRS-ESM) \\ and Department of Economics \\ Hamburg University \\ nicole.glanemann@wiso.uni-hamburg.de
}

January, 2012

\begin{abstract}
This paper examines the impact of Knightian uncertainty upon optimal climate policy through the prism of a continuous-time real option modelling framework. We analytically determine optimal intertemporal climate policies under ambiguous assessments of climate damages. Additionally, numerical simulations are provided to illustrate the properties of the model. The results indicate that increasing Knightian uncertainty accelerates climate policy, i.e. an ambiguity-averse policy maker become more reluctant to postpone the timing of climate policies into the future. However, we find that the size of this effect is rather small, which indicates that Knightian uncertainty is no "carte blanche" for extreme policy activism.
\end{abstract}

Keywords: Climate change, Knightian uncertainty, $\kappa$-ambiguity, real options JEL-Classification: C61, D81, Q54. 


\section{Introduction}

The future dynamics of greenhouse gas emissions, and their implications for global climate conditions in the future, will be shaped by the way in which policy makers respond to climate projections, react to model uncertainty, and derive resultant mitigation and adaptation decisions. However, assessments of the future impacts of climate change, which shall provide the basis of a climate policy decision, are far from being conclusive. A considerable lack of scientific understanding and uncertainties about the future economic development lead to enormous ambiguities in the projections. Hence, the question of how to design an optimal climate policy cannot be answered in an off-hand way. It could be reasonable to wait for new information to arrive before taking action. On the contrary, the peril of serious and irreversible climate damages may necessitate to take instantaneous and preventive action. This approach to the climate problem resembles the precautionary principle, which is for example endorsed by the European Union and the Rio Declaration on Environment and Development. Principle 15 in the 1992 Rio Declaration on Environment and Development states that due to threats of serious and irreversible damage, "lack of full scientific certainty shall not be used as a reason for postponing cost-effective measures to prevent environmental degradation."

To provide an economic foundation for this discussion, analysing the rational decision under fundamental uncertainties has gained importance over the last years. The lack of knowledge implies that the decision maker is not capable of assigning specific probabilities to events. Hence, the assumption of one probability measure, which is usually referred to as risk, falls short to capture the involved ambiguities. Due to the variety of possible scenarios, uncertainty is better described by a set of different probability measures. This concept, which is called Knightian uncertainty or ambiguity, captures uncertainty of the underlying probability measure, while risk means that uncertainty is only implied by the volatility of a known distribution. Examining how people react to situations of these different notions of uncertainty, Ellsberg (1961) provides experimental evidence that people usually prefer situations where probabilities are known. ${ }^{1}$ Accordingly, the decision maker is usually assumed to be ambiguity-averse. In this context, Gilboa \& Schmeidler (1989) give the axiomatic foundation that the set of probability distributions reduces to the behavioral bias to extreme pessimism, i.e. the decision maker maximises welfare of the minimum/worst scenario. Recently, this concept has been transfered to analyse the decision when to adopt an environmental policy. Asano (2010) examines the impacts of Knightian uncertainty referring to future economic developments that affect the social costs of a pollutant, e.g. the innovation of a technol-

\footnotetext{
${ }^{1}$ Before, it was widely accepted that a rational decision maker is considered to be indifferent between the situation where the probability is clear-cut and where it is vague, as long as the mean probability is the same for both cases, see Savage (1954). Subsequently, the so-called Ellsberg paradox has been examined and verified for alternative situations, see for example Becker \& Brownson (1964), Camerer \& Weber (1992), Halevy (2007), Hogarth \& Kunreuther (1985), Sarin \& Weber (1993) and Smith (1969). Neuro-empirical evidence has been provided by Hsu et al. (2005) by proving that certain areas in the brain respond differently to situations of risk and ambiguity.
} 
ogy could lower the costs of a climate policy adoption. Vardas \& Xepapadeas (2010) apply the Knightian uncertainty concept to the evolution of species biomass to assess ecosystem management strategies. These studies come to the same conclusion that the policy is to be adopted earlier than in a situation where uncertainty is described by risk. As pointed out by the authors, this approach can be considered to be a formal way to model the precautionary principle.

This paper reexamines this conclusion by providing a different view onto the effects of Knightian uncertainty. Different to the aforementioned papers, we direct the attention to the ambiguous assessments of the future damage costs. A review of the existing estimates reveals enormous uncertainties, see Stern (2007). Apart from different appraisals of vulnerabilities, impacts of extreme weather events and catastrophes are often neglected and underlying assumptions about the future economies' capability to adapt are highly controversial. Highlighting the ambiguity of these assessments, the three main benchmark studies by Mendelsohn et al. (2000), Nordhaus \& Boyer (2000) and Tol (2002) vary between 0 and 3 percent of GDP losses for a $3^{\circ} \mathrm{C}$ warming. Accordingly, we firstly develop a formal decision model where an ambiguity-averse social planner faces Knightian uncertainty in the future climate damage costs. We utilise numerical simulations to examine the timing of the policy adoption and to compare it to the situation where only risk exits. Secondly, we contribute to the literature by assessing the size of the effects caused by Knightian uncertainty. For this, the effects of marginal changes of the other crucial parameter values are chosen to serve as the basis for comparison. Is Knightian uncertainty as important to the optimal timing as the calibration of the discount rate and the abatement costs or does the ubiquitous parameter uncertainty swamp the effects by Knightian uncertainty? This kind of analysis contributes to a better understanding of decision making under ambiguity.

The model we develop is based on recent theoretical analyses of decisions under uncertainty, which have highlighted the effects of irreversibility in generating "real options". In these models, the interaction of time-varying uncertainty and irreversibility leads to a range of inaction where policy makers refer to "wait and see" rather than undertaking a costly action with uncertain consequences. We employ this recent literature and interpret climate policies as consisting of a portfolio of options. The general idea underpinning the view that climate policies are option-rights is that climate policy can be seen as analogous in its nature to the purchase of a financial call option, where the investor pays a premium price in order to get the right to buy an asset for some time at a predetermined price (exercise price), and eventually different from the spot market price of the asset. In this analogy, the policy maker, through her climate policy decision, pays a price which gives her the right to use a mitigation strategy, now or in the future, in return for lower damages. Taking into account this options-based approach, the calculus of suitability cannot be done simply applying the net present value rule, but rather has to consider the following three salient characteristics of the environmental policy decision: (i) there is uncertainty about future payoffs from cli- 
mate policies; (ii) waiting allows policy makers to gather new information on the uncertain future; and (iii) climate policies are at least partially irreversible. These characteristics are encapsulated in the concept of real option models. ${ }^{2}$ This strand of literature now constitutes a significant branch of the climate economics literature. A limited, but growing, strand of literature - particularly in mathematical economics - has extended the real options approach to analyse the interplay of irreversibility and uncertainty under Knightian uncertainty, see for example Nishimura \& Ozaki (2007) and Trojanowska \& Kort (2010). We transfer these ideas by expanding the paper by Pindyck (2009b) on uncertain outcomes and climate change policy.

The remainder of the paper is organised as follows. In Section 2, the comprehensive modelling set-up is presented. The framework incorporates cross-discipline interactions in order to derive dynamically optimal policy responses to Knightian uncertainty. Subsequently, in Section 3 we illustrate the working of the model through numerical exercises and examine the sensitivity of the main results with respect to key parameters. The paper concludes in Section 4 with a brief summary and suggestions for further research. Omitted details of several derivations are provided in appendices.

\section{The Model}

Over the last decades, climate models have been developed to an impressive level of complexity. Over a similar period, there has been growing interest in the uncertainty of future climate scenarios. Future climate projections are uncertain because both the initial conditions and the computational representation of the known equations of motion of the natural system are uncertain. To aid future climate policy decisions, accurate quantitative descriptions of the uncertainty in climate outcomes under various possible policies and scenarios are needed.

Of course, the multidisciplinary nature of the field presents a challenge. This requires integrating different natural and social sciences modelling paradigms traditions in a unified decision tool. Here, we have decided to extend the modelling framework of Pindyck $(2009 a, b)$ that embodies, in a simplified way, all essential ingredients by allowing for real options under Knightian uncertainty. The stochastic dynamic programming framework quantifies scientific uncertainties to the extent possible, and explains the potential implications of Knightian uncertainty for the outcomes of concern to the policy makers. It should be noted that the most obvious challenge along the way is to minimise complexity so that the model setup under complex uncertainty is still tractable. ${ }^{3}$

\footnotetext{
${ }^{2}$ Concise surveys of the real options literature are provided by Bertola (2010), Dixit \& Pindyck (1994) and Stokey (2009).

${ }^{3}$ The plethora of potentially significant contributions to overall atmospheric heat balance that are not treated in the simple model used here includes changes in other well-mixed greenhouse gases, ozone, snow albedo, cloud cover, solar irradiance, and aerosols. From this list, it should be clear that the objectives of the present paper are limited ones. A more complete assessment of outcome probabilities would include detailed
} 
The model assumes that a forward looking social planner strives to find the optimal timing of a climate policy by maximizing the flow of consumption over time. ${ }^{4}$ She faces the intergenerational trade-off problem that investments into a mitigation strategy, which substantially reduces emissions, force the present economy to abstain from consumption, but avoid climate damages that would decrease the future consumption potential. Moreover, a bad timing will certainly lead to one of the following two irreversibility effects. On the one hand, investing too early in mitigation technologies could trigger enormous sunk costs that are not recouped before long. On the other hand, waiting too long may cause irreversible damages to ecological systems that are valuable to human health or the economy. However, ubiquitous uncertainties in almost every component in the projections and especially in the assessment of future climate damages render a well-informed decision about the timing almost impossible. Put differently, all plans depend decisively on the unknown sensitivity of losses to climate change. Hence, particularly the uncertainties of the future climate damages and their effects are focussed on in the following, whereas any other lack of knowledge is assumed to be resolved for the sake of analytical tractability. Expressed mathematically, the policy maker solves the following isoelastic objective function, which consists of the expected net present value of future consumption levels:

$$
W=E\left[\int_{t=0}^{\infty} \frac{\left(L\left(X_{t}, \Delta \mathbb{T}_{t}\right) C_{t}\right)^{1-\delta}}{1-\delta} e^{-r t} d t\right]
$$

where $E[\cdot]$ is the expectation operator and $C_{t}$ is the aggregate consumption over time with the initial value normalised to 1 . In the simplest form, the level of consumption is assumed to be equivalent to the level of GDP. The parameter $\delta \geq 0$ is the inverse of the intertemporal elasticity of substitution and $r$ is the pure rate of social time preference. The climate damages are measured by $L\left(X_{t}, \Delta \mathbb{T}_{t}\right)$. This loss function is attached to the level of consumption, where $\Delta \mathbb{T}_{t}$ describes scientifically estimated changes in temperature and $X_{t}$ is a (positive) stochastic damage function parameter determining the sensitivity of losses to global warming.

Instead of trying to model climate impacts in any detail, we keep the problem analytically simple by assuming that damages depend only on the temperature change, which is chosen as a measure of climate change. To be precise, following Pindyck (2009a,b) we assume that the damage from warming and the associated physical impacts of climate change as a fraction of

models of the past and future of each of these effects.

${ }^{4}$ In our model framework we treat the world as a single entity in the interest of brevity. The world climate policy equilibrium can be constructed as a symmetric Nash equilibrium in mitigation strategies. The equilibrium can be determined by simply looking at the single country policy which is defined ignoring the other countries' abatement policy decisions [Leahy (1993)]. Furthermore, the modelling approach assumes that the social planner is benevolent. However, much discussion challenges this assumption. Climate policymakers, the argument goes, avoid hard decision, they pander the electorate, partisan politics leads to gridlock, and they do what is best for themselves. Discussing these flaws of policy decision making goes far beyond the scope of our research. 
GDP is implied by the exponential loss function

$$
L\left(X_{t}, \Delta \mathbb{T}_{t}\right)=e^{-X_{t}\left(\Delta \mathbb{T}_{t}\right)^{2}}
$$

where $0<L\left(X_{t}, \Delta \mathbb{T}_{t}\right) \leq 1, \partial L / \partial\left(\Delta \mathbb{T}_{t}\right) \leq 0$ and $\partial L / \partial X_{t} \leq 0$. This yields GDP at time $t$ net of damage from warming in the order of $L\left(X_{t}, \Delta \mathbb{T}_{t}\right) G D P_{t}$, i.e. climate-induced damages result in less GDP, and hence less consumption. ${ }^{5}$

Before we turn to the modelling of the uncertainty that is attached to $X_{t}$ in equation (2), we briefly introduce the other component in the loss function: the temperature increase $\Delta \mathbb{T}_{t}$. For this we adopt the commonly used climate sensitivity function in Weitzman (2009a) and Pindyck (2009a,b). The single linear differential equation compresses all involved complex physical processes by capturing climate forcings and feedbacks in a simplified manner. ${ }^{6}$ Hence, a direct link between the atmospheric greenhouse gas concentration $G_{t}$ and the temperature increase $\Delta \mathbb{T}_{t}$ is obtained by

$$
d \Delta \mathbb{T}_{t}=m_{1}\left(\frac{\ln \left(G_{t} / G_{0}\right)}{\ln 2}-m_{2} \Delta \mathbb{T}_{t}\right) d t
$$

where $G_{0}$ is the inherited pre-industrial baseline level of greenhouse gas, and $m_{1}$ and $m_{2}$ are positive parameters. The first term in the bracket stands for the radiative forcing induced by a doubling of the atmospheric greenhouse gases, i.e. $G_{t}$ is set to equal $2 G_{0}$. The second term represents the net of all negative and positive feedbacks. A positive parameter for this term thus rules out a runaway greenhouse effect. The parameter $m_{1}$ describes the thermal inertia or the effective capacity to absorb heat by the earth system, which is exemplified by the oceanic heat uptake.

By defining $H$ as the time horizon with $\Delta \mathbb{T}_{t}=\Delta \mathbb{T}_{H}$ at $t=H$ and $\Delta \mathbb{T}_{t} \rightarrow 2 \Delta \mathbb{T}_{H}$ as $t \rightarrow \infty$, we obtain equations, which are convenient to use in the real options setting. The following differential equations allow to derive the corresponding partial differential equation related to the real options terms and thus to solve the optimal stopping problem in a straightforward way:

$$
d \Delta \mathbb{T}_{t}=\frac{\ln (2)}{H}\left(2 \Delta \mathbb{T}_{H}-\Delta \mathbb{T}_{t}\right) d t,
$$

\footnotetext{
${ }^{5}$ Due the scarcity of empirical information about the magnitude of the damages in question, the shape of the damage function is somewhat arbitrary. Pindyck (2009b) has assumed the exponential function $L(\Delta \mathbb{T})=$ $\exp \left[-\beta\left(\Delta \mathbb{T}^{2}\right)\right]$, where $\beta$ follows a gamma distribution. This implies that future damages are fully captured by the probabilistic outcomes of a given distribution. This concept can be understood as risk. However, the present uncertainty about $\beta$ also comprises the choice of the probability distribution, which will be tackled in this paper.

${ }^{6}$ Factors that influence the climate are distinguished between forcings and feedbacks. A forcing is understood as a primary effect that changes directly the balance of incoming and outgoing energy in the earthatmosphere system. Emissions of aerosols and greenhouse gases or changes in the solar radiation are examples. A secondary and indirect effect is described by a feedback that boosts (positive feedback) or dampens (negative feedback) a forcing. The blackbody radiation feedback exemplifies an important negative feedback, whereas, for example, the ice-albedo feedback accelerates warming by decreasing the earth's reflectivity.
} 
and

$$
\Delta \mathbb{T}_{t}=2 \Delta \mathbb{T}_{H}\left(1-e^{-\frac{\ln 2}{H} t}\right)
$$

where $\ln (2) / H$ denotes the adjustment speed of changes in temperature to the eventual changes in temperature $2 \Delta \mathbb{T}_{H}{ }^{7}$

Let us now focus on the other component in equation (2), which is the sensitivity of losses to global warming. The standard real options approach emphasises the importance of uncertainty in determining option value and timing of option exercise. However, the standard real options approach rules out the situation where policy makers are unsure about the likelihoods of future events. It typically adopts strong assumptions about policy makers' beliefs and no distinction between risk and uncertainty is made. The usual prescription for decision making under risk then is to select an action that maximises expected utility. This is assumed although the knowledge of climate dynamics is still far from conclusive. ${ }^{8}$ New modelling techniques in natural science and greater computing power provide more details and finer distinctions, but do not necessarily lead to more accuracy in the projections. In the more realistic Knightian uncertainty scenario, policies therefore become more complex, as now the policy makers carry a set of probability measures for future climate change and consequently every policy measure is associated with an interval of expected costs. This implies that it would be more appropriate to describe the process of $X_{t}$ using a set of probability measures, not just one measure such as a geometric Brownian motion with a drift term as often used in real options. ${ }^{9}$ In other words, the Knightian version of the real options models differs from the plain vanilla real options model by having an entire set of subjective probability distributions. Modelling Knightian uncertainty is a non-trivial task in general. To incorporate a situation where policy makers are unable to assign a precise probability to future alternatives, we use the Knightian uncertainty modelling approach developed by Nishimura \& Ozaki (2007). In their comprehensive representation of Knightian uncertainty, unresolved processes are represented by computationally efficient stochastic-dynamic schemes. We introduce their

\footnotetext{
${ }^{7}$ There is considerable a priori uncertainty in the probability and scale of climate change, but at least there are historical time series data available to calibrate probability distributions for parameters important in modelling climate sensitivity. On the other hand, based on current knowledge there is a large a priori uncertainty concerning when dramatic technological breakthroughs might occur and how much impact they will have, so allowing for such possibilities should increase the spread of outcomes for global carbon emissions and their consequences.

${ }^{8}$ One has to admit that despite more observations, more sophisticated coupled climate models and substantial increases in computing power, climate projections have not narrowed appreciably over the last two decades. Indeed, it has been speculated that foreseeable improvements in the understanding of the underlying physical processes will probably not lead to large reductions in climate sensitivity uncertainty. See Roe \& Baker (2007).

${ }^{9}$ Alternatively, the imprecise probability concept in Reichert (1997) employs a set of probability measures describing the uncertain model parameters. For example, the ambiguity involved in the estimation of the global mean temperature change in the 21st century is analysed in Kriegler \& Held (2005) by constructing a belief function that is the lower envelope of the corresponding distributions. The model results in large imprecisions of the estimates, highlighting the key role of uncertainties in climate projections. Apart from deriving upper and lower bounds of the sets, Borsuk \& Tomassini (2005) examine other representations of the probability measures and demonstrate how to use them to describe climate change uncertainties.
} 
treatment of Knightian uncertainty below.

To formalise the concept, let $\left(B_{t}\right)_{0 \leq t \leq T}$ be a standard Brownian motion on $\left(\Omega, \mathscr{F}_{T}, P\right)$ that is endowed with the standard filtration $\left(\mathscr{F}_{t}\right)_{0 \leq t \leq T}$ for $\left(B_{t}\right)$. Consider the real-valued stochastic process $\left(X_{t}\right)_{0 \leq t \leq T}$ generated by the Brownian motion with drift $\alpha$ and standard deviation $\sigma$ :

$$
d X_{t}=\alpha X_{t} d t+\sigma X_{t} d B_{t}
$$

In equation (6) the particular probability measure $P$ is regarded as capturing the true nature of the underlying process. ${ }^{10}$ This, however, is highly unlikely, as this would imply that policy makers are absolutely certain about the probability distribution that describes the future development of $\left(X_{t}\right)_{0 \leq t \leq T}$. Unlike this standard case, Knightian uncertainty describes how policy makers form ambiguous beliefs. Thereby a set $\mathscr{P}$ of probability measures is assumed to comprise likely candidates to map the future dynamics.

Technically spoken, these measures are generated from $P$ by means of density generators, $\theta .{ }^{11}$ Such a probability measure is denoted by $Q^{\theta}$ in the following. By restricting the density generators to a certain range like a real-valued interval $[-\kappa, \kappa]$, we are enabled to confine the range of deviations from the original measure $P$. The broader this interval is, the larger the set of probability measures, $\mathscr{P}=\left\{Q^{\theta} \mid \theta \in[-\kappa, \kappa]\right\}$, and thus the higher the degree of ambiguity. This specific notion of confining the density generators to an interval $[-\kappa, \kappa]$ is named $\kappa$-ignorance by Chen \& Epstein (2002), who have applied this to a different field of research.

Endowed with this concept we can now define a stochastic processes $\left(B_{t}^{\theta}\right)_{0 \leq t \leq T}$ by

$$
B_{t}^{\theta}=B_{t}+t \theta
$$

for each $\theta \in[-\kappa, \kappa]$. As Girsanov's theorem shows, each process $\left(B_{t}^{\theta}\right)_{0 \leq t \leq T}$ defined as above is a standard Brownian motion with respect to $Q^{\theta}$ on $\left(\Omega, \mathscr{F}_{T}, Q^{\theta}\right)$. Inserting the definition of $\left(B_{t}^{\theta}\right)_{0 \leq t \leq T}$ into equation (6), we obtain for every $\theta \in[-\kappa, \kappa]$

$$
d X_{t}=(\alpha-\sigma \theta) X_{t} d t+\sigma X_{t} d B_{t}^{\theta}
$$

\footnotetext{
${ }^{10}$ The Brownian motion in equation (6) is a reasonable approximation and we share this assumption with most of the existing literature.

${ }^{11}$ Assume a stochastic process $(\theta)_{0 \leq t \leq T}$ that is real-valued, measurable and $\left(\mathscr{F}_{t}\right)$-adapted. Furthermore it is twice integrable, hence $\theta:=(\theta)_{0 \leq t \leq \mathcal{T}} \in \mathcal{L}^{2} \subset \mathcal{L}$. Define $\left(z_{t}^{\theta}\right)_{0 \leq t \leq T}$ by $z_{t}^{\theta}=e^{\left(-\frac{1}{2} \int_{0}^{t} \theta_{s}^{2} d s-\int_{0}^{t} \theta_{s} d B_{s}\right)} \quad \forall t \geq 0$. Note that the stochastic integral $\int_{0}^{t} \theta_{s} d B_{s}$ is well-defined for each $t$, as $\theta \in \mathcal{L}$. A stochastic process $\theta \in \mathcal{L}$ is a density generator, if $\left(z_{t}^{\theta}\right)_{0 \leq t \leq T}$ is a $\left(\mathscr{F}_{t}\right)$-martingale. Using a density generator $\theta$ another probability measure $Q^{\theta}$ on $\left(\Omega, \mathscr{F}_{T}\right)$ can be generated from $P$ by

$$
Q^{\theta}(A)=\int_{A} z_{T}^{\theta} d P \quad \forall A \in \mathscr{F}_{T}
$$
}

Note that any probability measure that is thus defined is called equivalent to $P$. 
Equation (8) displays all stochastic differential equations and thus all future developments of $\left(X_{t}\right)_{0 \leq t \leq T}$ that the decision maker thinks possible. Note that the implementation of Knightian uncertainty implies different drift but the same volatility terms.

One element in the above generated set of stochastic differential equations is of special importance. Ellsberg (1961) provides experimental evidence that people usually prefer situations where probabilities are known. Hence, it is reasonable to assume that the policy maker is ambiguity-averse. Gilboa \& Schmeidler (1989) prove that ambiguity-aversion makes the decision maker maximize the worst case scenario. As $e^{-X_{t}\left(\Delta \mathbb{T}_{t}\right)^{2}} G D P_{t}$ is calculated as GDP net of damages, the worst case scenario is described by the largest value of $X_{t}$. As an illustration and in order to gain an intuition we have numerically simulated equation (2) and (6) for a time period of 200 years for $\Delta \mathbb{T}_{H}=1.9^{\circ} \mathrm{C}$ versus $\Delta \mathbb{T}_{H}=3.4^{\circ} \mathrm{C}$ (equivalent to pre-industry levels of $2.5^{\circ} \mathrm{C}$ versus $4^{\circ} \mathrm{C}$ ) of warming and three alternative drift terms. The character of the impact function (2) for various drift terms is shown in Figure 1. The various graphs indicate the forces at play in our analysis. Two effects must be recognised. First, the highest value of the drift term generates the maximum of $1-L\left(X_{t}, \Delta \mathbb{T}_{t}\right)$ and therefore the minimum of $G D P_{t}$ net of damages. Second, as can be seen the function $L\left(X_{t}, \Delta \mathbb{T}_{t}\right)$ spreads out considerably for higher temperature increases. After 100 years and for $\Delta \mathbb{T}=3.4^{\circ} \mathrm{C}$ the damage is $0.09154=9.15$ percent of GDP. ${ }^{12}$

After understanding the process of $X_{t}$, we can now discuss the optimal response to climate change under Knightian uncertainty. If the ambiguity-averse decision maker conducts no climate policy - referred to as the business as usual approach - and faces Knightian uncertainty in equation (1), then the resulting intertemporal welfare, $W^{\mathrm{N}}$, with consumption growing at a rate $g_{0}$ and initial consumption normalised as 1 is determined as

$$
\begin{aligned}
W^{\mathrm{N}}\left(X, \Delta \mathbb{T} ; \Delta \mathbb{T}_{H}\right) & =\min _{Q^{\theta} \in \mathscr{P}} E^{Q^{\theta}}\left[\int_{t=0}^{\infty} \frac{\left(e^{\left.-X_{s}\left(\Delta \mathbb{T}_{s}\right)^{2} C_{s}\right)^{1-\delta}} e^{-r s} d s \mid \mathscr{F}_{t}\right]}{1-\delta}\right. \\
& =\frac{1}{1-\delta} \min _{Q^{\theta} \in \mathscr{P}} E^{Q^{\theta}}\left[\int_{t=0}^{\infty} e^{-X_{s}(1-\delta)\left(\Delta \mathbb{T}_{s}\right)^{2}} e^{-\left(r-(1-\delta) g_{0}\right) s} d s \mid \mathscr{F}_{t}\right],
\end{aligned}
$$

s.t. equations (4) and (8), where "N" refers to the no-actions-taken approach, $r-(1-\delta) g_{0}$ is assumed to be positive, and $E^{Q^{\theta}}\left[\cdot \mid \mathscr{F}_{t}\right]$ represents the expectation value with respect to $Q^{\theta} \in \mathscr{P}$ conditional on $\mathscr{F}_{t} .{ }^{13}$ The first equation holds as ambiguity aversion implies that the

\footnotetext{
${ }^{12}$ The calibrated damages from warming are in the range of previous estimates. Weitzman (2009b) has assumed damage costs of 1.7 percent of GDP for $2.5^{\circ} \mathrm{C}$ of warming - a level that is considered to be a threshold for danger. For higher temperature increases he has assumed rapidly increasing damages of 9 (25) percent of GDP for $4^{\circ} \mathrm{C}\left(5^{\circ} \mathrm{C}\right)$ of warming. Millner et al. (2010) have assumed damages of $1.7(6.5)$ percent of GDP for $2.5^{\circ} \mathrm{C}\left(5^{\circ} \mathrm{C}\right)$ of warming.

${ }^{13}$ For reasons of mathematical tractability we assume that the continuous Knightian uncertainty is independent of time and therefore the planning horizon is infinite. The reasoning for the perpetual assumption is that the underlying time scales in the natural climate system are much longer than those in the economic system. Technically, we consider $T \rightarrow \infty$ for $\left(B_{t}\right)_{0 \leq t \leq T}$ and $\left(B_{t}^{\theta}\right)_{0 \leq t \leq T}$ in the above made introduction to the concept of Knightian uncertainty.
} 

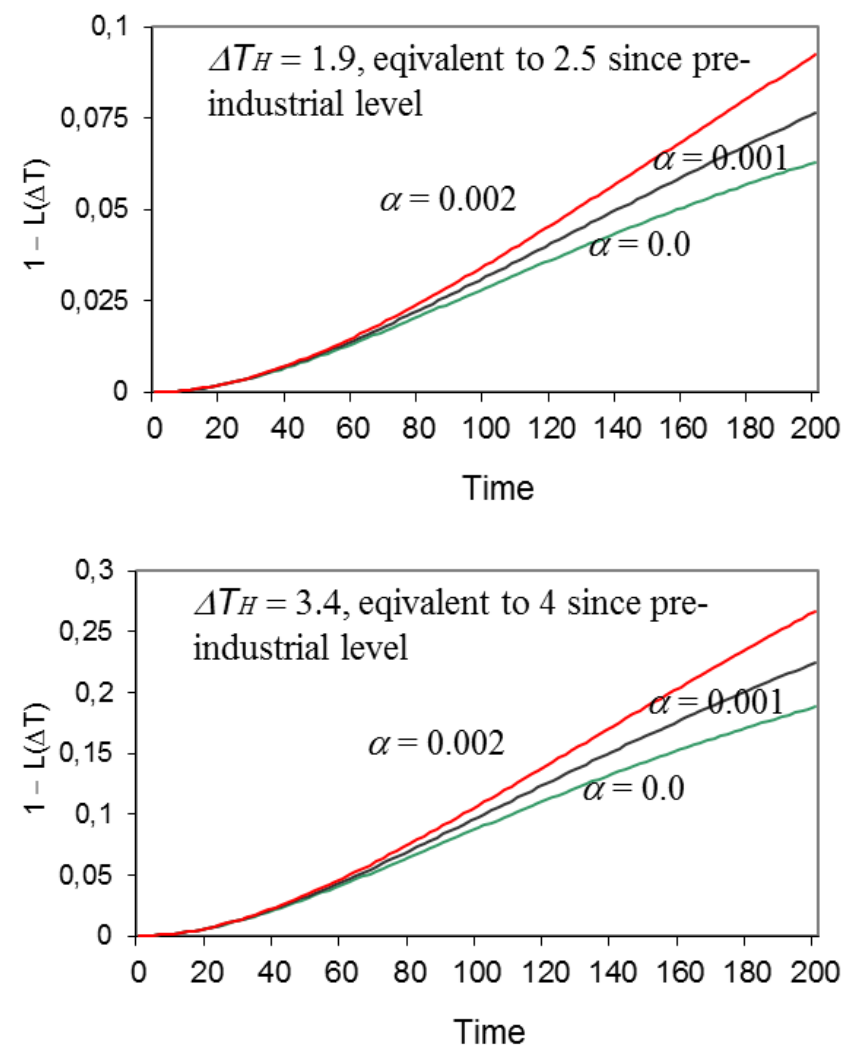

Figure 1: Simulated Damages $1-L\left(X_{t}, \Delta \mathbb{T}_{t}\right)$ Due To Global Warming in Percent of GDP. The initial value for $X$ is $X_{0}=0.008$ and $H=100$. The simulated time series are computed ignoring the uncertainty part of equation (6), i.e. $d X_{t}=\alpha X_{t} d t$.

policy maker reckons with the lowest expected welfare value. ${ }^{14}$ Please note that the impact of Knightian uncertainty is not necessarily monotonous for the policy maker. As shown in the following, $W^{\mathrm{N}}$ consists of two components: a particular integral that expresses the perpetual business as usual policy and the real options to adopt the policy. Both parts are affected by the Knightian uncertainty. ${ }^{15}$

For the sake of analytical tractability, we apply a Taylor series expansion to $e^{-X_{s}(1-\delta) \Delta \mathbb{T}_{s}^{2}}$ such that

$$
e^{-X_{s}(1-\delta) \Delta \mathbb{T}_{s}^{2}} \cong 1-X_{s}(1-\delta) \Delta \mathbb{T}_{s}^{2}+\frac{1}{2}\left(X_{s}(1-\delta) \Delta \mathbb{T}_{s}^{2}\right)^{2},
$$

where $0<L\left(\Delta \mathbb{T}_{t}\right) \leq 1$ and $\partial L / \partial\left(\Delta \mathbb{T}_{t}\right) \leq 0$ still hold. ${ }^{16}$ By inserting (10) into (9) we thus

\footnotetext{
${ }^{14}$ First, the ambiguity-averse policy maker takes only the probability measure into consideration that creates the worst outcomes for the welfare. Then she strives to find the policy strategy that maximises this 'worstcase welfare function'. The maxmin nature of the problem links the analysis with contributions on robust control. See, for example, Funke \& Paetz (2011).

${ }^{15}$ Though real options only dominate the particular integral with extreme Knightian uncertainty, while the effect of smaller Knightian uncertainty on the particular integral is prevailing.

${ }^{16}$ Real option models suggested in the literature seem always to make a trade-off between analytical tractability and realism. In this paper we analyse a model that combines both features into one model: the model has a rich analytical structure and nevertheless the analytical forms of the particular solutions can be obtained. The approximation appears from simulations to be a good proxy. The numerical simulations
} 
obtain

$$
\begin{aligned}
& W^{\mathrm{N}}\left(X, \Delta \mathbb{T} ; \Delta \mathbb{T}_{H}\right) \\
& =\frac{1}{1-\delta} \min _{Q^{\theta} \in \mathscr{P}} E^{Q^{\theta}}\left[\int_{t=0}^{\infty}\left(1-X_{s}(1-\delta) \Delta \mathbb{T}_{s}^{2}+\frac{1}{2}\left(X_{s}(1-\delta) \Delta \mathbb{T}_{s}^{2}\right)^{2}\right) e^{-\left(r-(1-\delta) g_{0}\right) s} d s \mid \mathscr{F}_{t}\right],
\end{aligned}
$$

s.t. equation (4) and (8). Using Itô's Lemma and following the standard dynamic programming argument, we formulate the problem in terms of the Hamilton-Jacobi-Bellman equation $^{17}$

$$
\begin{aligned}
\left(r-(1-\delta) g_{0}\right) W^{\mathrm{N}}=\frac{1}{1-\delta}-X^{*} \Delta \mathbb{T}^{2}+\frac{1}{2} X^{*^{2}}(1-\delta) \Delta \mathbb{T}^{4} \\
+\frac{\ln (2)}{H}\left(2 \Delta \mathbb{T}_{H}-\Delta \mathbb{T}\right) \frac{\partial W^{\mathrm{N}}}{\partial \Delta \mathbb{T}}+(\alpha+\kappa \sigma) X^{*} \frac{\partial W^{\mathrm{N}}}{\partial X^{*}}+\frac{1}{2} \sigma^{2} X^{2} \frac{\partial^{2} W^{\mathrm{N}}}{\partial X^{*^{2}}}
\end{aligned}
$$

The asterisk represents the density generator $-\kappa$, meaning that $Q^{*}$ is generated by $-\kappa$ and the stochastic process $X^{*}$ is defined by inserting $-\kappa$ into equation (8):

$$
d X_{t}^{*}=(\alpha+\sigma \kappa) X_{t}^{*} d t+\sigma X_{t}^{*} d B_{t}^{-\kappa}
$$

For policies to be optimal, equation (12) must hold.

The solution of equation (12) is the sum of a particular and general solution. The particular solution $W^{\mathrm{NP}}$ is obtained by integrating the integral for $W^{\mathrm{N}}$ of equation (11) without considering possible policy intervention. Therefore, the real options terms are not exercised. It is straightforward to explain $W^{\mathrm{NP}}$ as the value of the business as usual policy. The policy maker does not intervene through exercising the real options to reduce the green house gas emissions, which leads to a cap of the future temperature changes $\Delta \mathbb{T}_{H}$. The general/homogeneous solutions or real options solutions $W^{\mathrm{NG}}$ are obtained by focusing attention on the homogeneous part of equation (12) such that

$$
\begin{aligned}
\left(r-(1-\delta) g_{0}\right) W^{\mathrm{NG}} & =\frac{\ln (2)}{H}\left(2 \Delta \mathbb{T}_{H}-\Delta \mathbb{T}\right) \frac{\partial W^{\mathrm{NG}}}{\partial \Delta \mathbb{T}} \\
& +(\alpha+\kappa \sigma) X^{*} \frac{\partial W^{\mathrm{NG}}}{\partial X^{*}}+\frac{1}{2} \sigma^{2} X^{*^{2}} \frac{\partial^{2} W^{\mathrm{NG}}}{\partial X^{*^{2}}}
\end{aligned}
$$

Now we turn our attention to the welfare value of implementing a climate policy. Let us assume that the policy maker is willing to pay annual abatements costs $w(\tau)$ as a percentage of GDP to limit the temperature increase at $t=H$ to less than or equal to $\tau: \Delta \mathbb{T}_{H} \leq \tau .{ }^{18}$

we conduct in the next section show that the thresholds $X$ are around 0.012 by the benchmark values, which implies that the scale of the 2 nd order term for $X$ is relatively small $\left(0.5 \cdot 0.012^{2}=0.000072\right)$, compared with the first order term.

${ }^{17}$ For the derivation please see Appendix A.

${ }^{18}$ In practical terms, this means that the policy maker reduces $G_{t}$ in equation (3) so that the increase in 
Analogous to the derivation procedure in Appendix A, the intertemporal welfare function of taking action to reduce the green house gas emission, $W^{\mathrm{A}}$, is then given by

$$
\begin{aligned}
\left(r-(1-\delta) g_{0}\right) & W^{\mathrm{A}}=(1-w(\tau))^{1-\delta}\left(\frac{1}{1-\delta}-X^{*} \Delta \mathbb{T}^{2}+\frac{1}{2} X^{*^{2}}(1-\delta) \Delta \mathbb{T}^{4}\right) \\
+ & \frac{\ln (2)}{H}(2 \tau-\Delta \mathbb{T}) \frac{\partial W^{\mathrm{A}}}{\partial \Delta \mathbb{T}}+(\alpha+\kappa \sigma) X^{*} \frac{\partial W^{\mathrm{A}}}{\partial X^{*}}+\frac{1}{2} \sigma^{2} X^{*^{2}} \frac{\partial^{2} W^{\mathrm{A}}}{\partial X^{*^{2}}}
\end{aligned}
$$

which is derived from the following integral

$W^{\mathrm{A}}(t=0, X, \Delta \mathbb{T} ; \tau)=\frac{1}{1-\delta} \times$

$E^{Q^{*}}\left[(1-w(\tau))^{1-\delta} \int_{t=0}^{\infty}\left(1-X_{s}^{*}(1-\delta) \Delta \mathbb{T}_{s}^{2}+\frac{1}{2}\left(X_{s}^{*}(1-\delta) \Delta \mathbb{T}_{s}^{2}\right)^{2}\right) e^{-\left(r-(1-\delta) g_{0}\right) s} d s \mid \mathscr{F}_{t}\right]$,

s.t. equation (8), and equation (17) that is

$$
d \Delta \mathbb{T}_{s}=\frac{\ln (2)}{H}\left(2 \tau-\Delta \mathbb{T}_{s}\right) d s
$$

where equation (17) is a variant of equation (4) by replacing $\Delta \mathbb{T}_{H}$ with $\tau$. If climate policy is time-consistent, then the solutions to $W^{\mathrm{A}}$ can be obtained by integrating equation (17) directly. In this case, the thresholds for $X^{*}$ of taking actions to limit the future temperature increase to less than or equal to $\tau$ at $t=H$ are then computed from the identity

$$
W(\text { taking action })=W(\text { business as usual })+\text { Real options. }
$$

Substituting, we have

$$
W^{\mathrm{A}}(\bar{X}, \Delta \mathbb{T} ; \tau)=W^{\mathrm{NP}}\left(\bar{X}, \Delta \mathbb{T} ; \Delta \mathbb{T}_{H}\right)+W^{\mathrm{NG}}\left(\bar{X}, \Delta \mathbb{T} ; \Delta \mathbb{T}_{H}\right)
$$

where $\bar{X}$ denotes the thresholds at which the policy-maker would take action by exercising the real options today and committing paying annual abatement $\operatorname{costs} w(\tau)$ in percent of GDP to limit the future temperature increase to less than $\tau$ at $t=H$. On the contrary, exercising of the real options $W^{\mathrm{NG}}\left(\bar{X}, \Delta \mathbb{T} ; \Delta \mathbb{T}_{H}\right)$ implies that the policy maker forgoes the option to wait and act later as more information about $X_{t}$ becomes available.

The next step is to solve the particular integrals of $W^{\mathrm{NP}}$ and $W^{\mathrm{A}}$, and real options $W^{\mathrm{NG}}$. As there are no uncertain terms for the processes of changes in temperatures $\Delta \mathbb{T}_{t}$, we can use equation (5) to obtain

$$
\Delta \mathbb{T}_{t}=2 \tau\left(1-e^{-\frac{\ln 2}{H} t}\right) .
$$

$\overline{\text { temperature is limited to less than } \tau \text { at } t=H}$. 
As shown in Appendix B the following particular integrals result from Itô's Lemma:

$$
W^{\mathrm{NP}}\left(X, \Delta \mathbb{T} ; \Delta \mathbb{T}_{H}\right)=\frac{1}{1-\delta}\left[\frac{1}{r-(1-\delta) g_{0}}-4 \Delta \mathbb{T}_{H}^{2}(1-\delta) \gamma_{1} X^{*}+8 \Delta \mathbb{T}_{H}^{4}(1-\delta)^{2} \gamma_{2} X^{*^{2}}\right]
$$

$$
W^{\mathrm{A}}(X, \Delta \mathbb{T} ; \tau)=\frac{(1-w(\tau))^{1-\delta}}{1-\delta}\left[\frac{1}{r-(1-\delta) g_{0}}-4 \Delta \tau^{2}(1-\delta) \gamma_{1} X^{*}+8 \Delta \tau^{4}(1-\delta)^{2} \gamma_{2} X^{*^{2}}\right]
$$

where

$$
\begin{aligned}
& \gamma_{1}=\frac{1}{\eta_{1}}-\frac{2}{\eta_{1}+\frac{\ln 2}{H}}+\frac{1}{\eta_{1}+2 \frac{\ln 2}{H}}, \\
& \gamma_{2}=\frac{1}{\eta_{2}}-\frac{4}{\eta_{2}+\frac{\ln 2}{H}}+\frac{6}{\eta_{2}+2 \frac{\ln 2}{H}}-\frac{4}{\eta_{2}+3 \frac{\ln 2}{H}}+\frac{1}{\eta_{2}+4 \frac{\ln 2}{H}}, \\
& \eta_{1}=r-(1-\delta) g_{0}-(\alpha+\kappa \sigma), \\
& \eta_{2}=r-(1-\delta) g_{0}-\left(2(\alpha+\kappa \sigma)+\sigma^{2}\right) .
\end{aligned}
$$

Note that it is assumed that both $\eta_{1}$ and $\eta_{2}$ are positive.

After obtaining the analytical particular solutions of equations (21) and (22), we now need to turn our attention to the real options term $W^{\mathrm{NG}}$ in equation (14). In Appendix C we show that the general solutions have the forms:

$$
W^{\mathrm{NG}}\left(t=0, X, \Delta \mathbb{T} ; \Delta \mathbb{T}_{H}\right)=A_{1} X^{*^{\beta_{1}}}\left(\Delta \mathbb{T}^{2}-4 \Delta \mathbb{T}_{H} \Delta \mathbb{T}+4 \Delta \mathbb{T}_{H}^{2}\right),
$$

where $\beta_{1}$ is the positive root of the quadratic characteristic equation

$$
\frac{1}{2} \sigma^{2} \beta(\beta+1)+(\alpha+\kappa \sigma) \beta-\left(r-(1-\delta) g_{0}+2\left(\frac{\ln (2)}{H}\right)\right)=0,
$$

and $A_{1}$ is the unknown parameter to be determined by the value-matching and smoothpasting conditions. The meaning of equation (23) is straightforward. For a small $\Delta \mathbb{T}_{H}$ the value of the options to take actions is small - the option of taking action is reduced for less global warming. The effective discount rate for real options is a positive function of $2 \ln (2) / H$. As we know from equation $(4), \ln (2) / H$ also denotes the adjustment speed of changes in temperature. Higher adjustment speed to the higher temperature (for example, $H=50$ years instead of $H=100$ years) means that the damage is higher and thus the option value is smaller. After obtaining the solutions to equation (19) by applying the valuematching condition, the smooth-pasting condition is given by equalising the derivative of (22) with respect to $X^{*}$ with the sum of the derivatives of (21) and (23) with respect to $X^{*}$. 
Substituting (21) - (23) back into the value-matching and smooth-pasting conditions yields

$$
\begin{array}{r}
4 \gamma_{1}\left(\Delta \mathbb{T}_{H}^{2}-\Delta \tau^{2}(1-w(\tau))^{1-\delta}\right) \bar{X}^{*}-8(1-\delta) \gamma_{2}\left(\Delta \mathbb{T}_{H}^{2}-(1-w(\tau))^{1-\delta} \tau^{4}\right) \bar{X}^{*^{2}} \\
=\frac{1-(1-w(\tau))^{1-\delta}}{\left(r-(1-\delta) g_{0}\right)(1-\delta)}+A_{1} \bar{X}^{*^{\beta_{1}}}\left(\Delta \mathbb{T}^{2}-4 \Delta \mathbb{T}_{H} \Delta \mathbb{T}+4 \Delta \mathbb{T}_{H}^{2}\right),
\end{array}
$$

and

$$
\begin{aligned}
& 4 \gamma_{1}\left(\Delta \mathbb{T}_{H}^{2}-\Delta \tau^{2}(1-w(\tau))^{1-\delta}\right)- 16(1-\delta) \gamma_{2}\left(\Delta \mathbb{T}_{H}^{2}-(1-w(\tau))^{1-\delta} \tau^{4}\right) \bar{X}^{*} \\
&=A_{1} \beta_{1} \bar{X}^{*_{1}-1}\left(\Delta \mathbb{T}^{2}-4 \Delta \mathbb{T}_{H} \Delta \mathbb{T}+4 \Delta \mathbb{T}_{H}^{2}\right) .
\end{aligned}
$$

So far, our discussion of Knightian uncertainty has been exclusively analytical. With the optimality conditions and the value-matching and smooth-pasting conditions, we can now proceed to the numerical simulations of the model.

\section{$3 \quad$ Numerical Simulations and Results}

While the preceding section has laid out the modelling framework, we now focus on a thorough numerical analysis of the model. Firstly, we aim to clarify whether the more realistic assumption of ambiguity implies a timing of policy adoption that is different from the one in the usually applied approach of risk. Secondly, our simulations illustrate the magnitude of the effects in terms of changes in the threshold of taking action. Note, however, that the goal of this paper is not to derive precise quantitative estimates of the impact of Knightian uncertainty, but rather to illustrate the scale of the Knightian uncertainty impact, and to see what we can learn from this framework. For example, we may find out the sensitivity of how the model reacts to the choice of $\kappa$, which is problematic to quantify. More precisely, the sensitivity to $\kappa$ is compared with the sensitivity to the other parameters whose calibration is up for debate. The baseline calibration of these parameters requires the use of judgement, i.e. they reflect a back-of-the-envelope calculation. ${ }^{19}$

The unit time length corresponds to one year. Our base parameters are $\sigma=0.075, \kappa=0.02$, $r=0.04, \alpha=0.0, g_{0}=0.01, \delta=0.0$, and $H=100 . \Delta \mathbb{T}_{H}$ is assumed to be $3.4^{\circ} \mathrm{C}$ which is equivalent to 4 degrees of warming since the pre-industrial level. $\tau$ is assumed to be $1.4^{\circ} \mathrm{C}$ by assumption which is equivalent to 2 degrees of warming compared with the pre-industrial level. Special attention has to be paid to the calibration of $w(\tau)$. The term $w(\tau)$ represents the achievability and costs of climate targets. What are the economic costs of reaching the target of climate stabilisation at no more than $2^{\circ} \mathrm{C}$ above pre-industrial level by the end of this century? To assess this question, Edenhofer et al. (2010) have compared the energy-

\footnotetext{
${ }^{19}$ Despite the increasingly detailed understanding of climate processes from a large body of research, various parameters involved inevitably remain inestimable, except in retrospect. Moreover, the calibrated model is not based on detailed time series data in the way econometric models are and does not have the projective power of the latter.
} 
environment-economy models MERGE, REMIND, POLES, TIMER and E3MG in a model comparison exercise. In order to improve model comparability, the macroeconomic drivers in the five modelling frameworks employed were harmonised to represent similar economic developments. On the other hand, different views of technology diffusion and different structural assumptions regarding the underlying economic system across the models remained. This helps to shed light on how different modelling assumptions translate into differences in mitigation costs. Low stabilisation crucially depends upon learning and technologies available. Despite different structures employed in the models, four of the five models show a similar pattern in mitigations costs for achieving the first-best $400 \mathrm{ppm} \mathrm{CO}_{2}$ concentration pathway. After allowing for endogenous technical change and carbon capture and storage with a storage capacity of at least $120 \mathrm{GtC}$, the mitigation costs are estimated to be approximately 2 percent of worldwide GDP. These costs turned out to be of a similar order of magnitude across the models. We therefore assume that $w(\tau)=0.02$.

In the real option literature the problem we must solve is referred to as "optimal stopping". The idea is that at any point in time the value of temperature reductions is compared with the expected value of waiting $d t$, given the available information set and the knowledge of the stochastic processes. First, we consider the thresholds for adopting climate policies, i.e. we calculate the optimal timing of adopting climate policies. The optimal strategy is to stop and adopt the climate policy right now if $X_{t}^{*} \geq \bar{X}^{*}$ and to continue waiting if $X_{t}^{*}<\bar{X}^{*}$, where $\bar{X}^{*}$ is the threshold value. ${ }^{20}$ To start with, in Figures 2 and 3 we focus our attention on the sensitivity of the optimal thresholds of an ambiguity-averse policy maker with respect to the degree of Knightian uncertainty $\kappa$ and changes in the degree of risk, i.e. the volatility of the geometric Brownian motion process $\sigma$. The solutions for $\kappa=0$ characterise the situation of a single probability measure and therefore the situation without Knightian uncertainty as in a traditional real option framework.

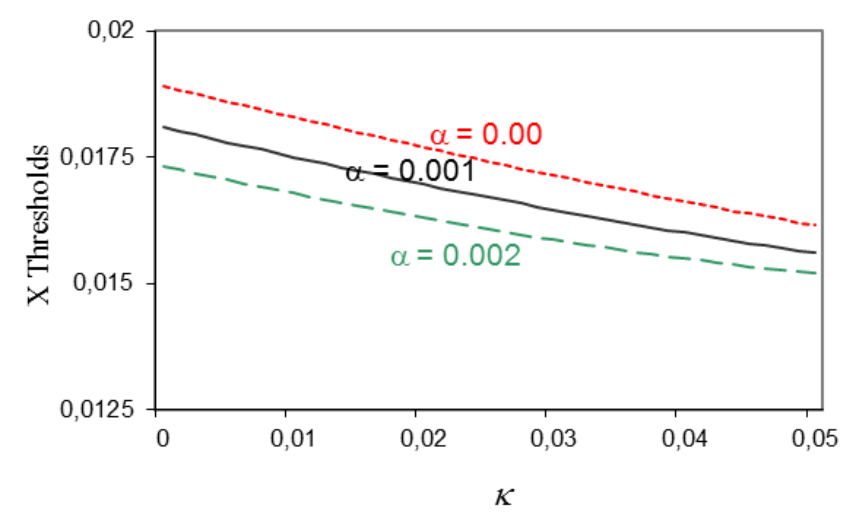

Figure 2: The Climate Policy Thresholds for Alternative $\kappa$ 's and $\alpha$ 's

\footnotetext{
${ }^{20}$ It is worth conjecturing that the existence of the no action area sheds light on why policy makers often deem it desirable to stay put, contrary to intuition which stems from thinking in terms of a simple cause and effect framework.
} 
Figure 2 provides a sensitivity analysis of the threshold with respect to $\kappa$. The numerical results indicate an acceleration of climate policy for higher degrees of Knightian uncertainty, i.e. increasing ambiguity has an unequivocally positive impact upon the timing of optimal climate policy and shrinks the continuation region where exercising climate policy is suboptimal. Hence, this result for the special case of ambiguity in the damage costs is in line with the literature that examines Knightian uncertainty in other components of an economic climate model, see Asano (2010) and Vardas \& Xepapadeas (2010). Figure 2 also shows that this result is insensitive to the choice of the drift parameter $\alpha$, which can be regarded as a measure of the economy's vulnerability. The higher $\alpha$ is, the more vulnerable the economy over time. Therefore it is clear, that a higher vulnerability implies a decrease of the threshold.
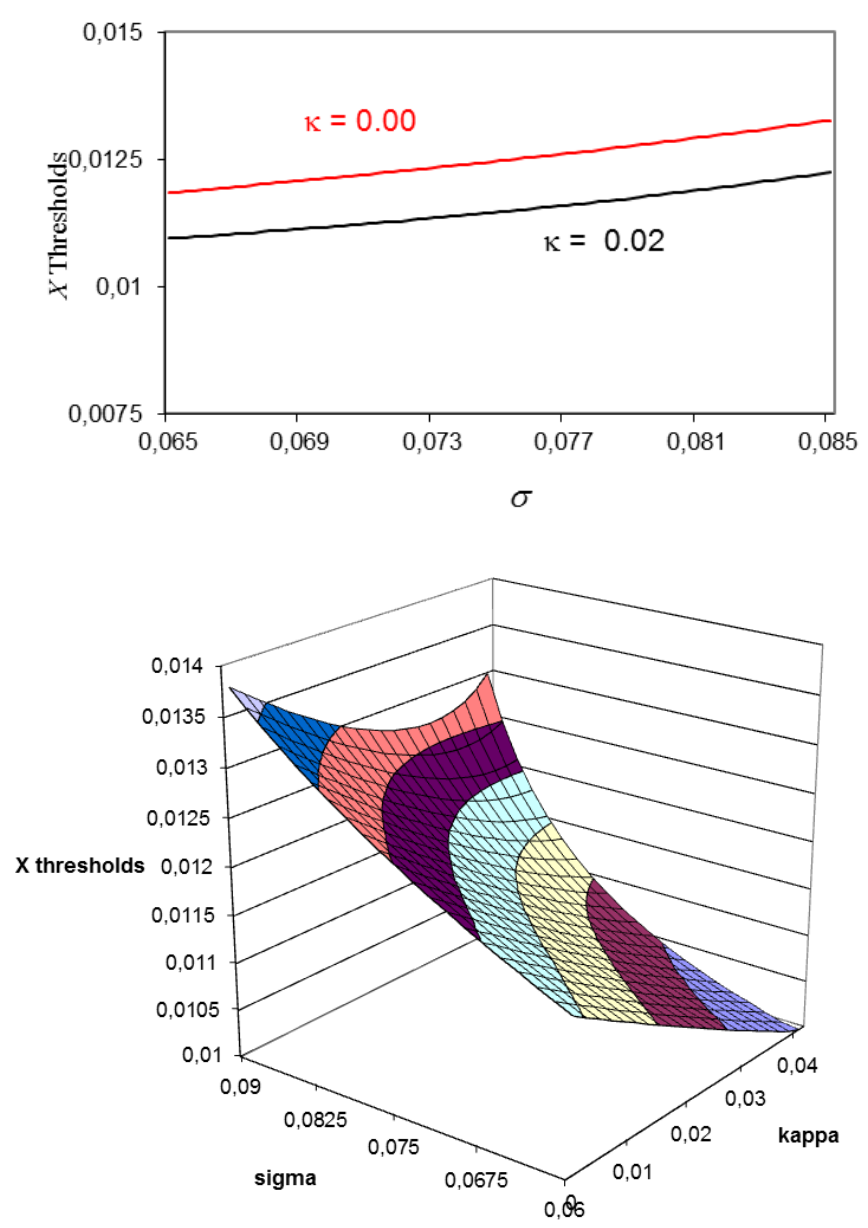

Figure 3: The Climate Policy Thresholds for Alternative $\sigma$ 's and $\kappa$ 's

In contrast, Figure 3 indicates that the threshold value at which climate policy is implemented is increasing in the noisiness level $\sigma$. The intuition is that the policy maker can counteract the impact from additional risk by a wait and see attitude for the time being. The case $\kappa=$ 0 again represents the case of no Knightian uncertainty. As expected, increased Knightian uncertainty tends to accelerate optimal timing, while increased risk $\sigma$ leads to the opposite response. Additional observations concerning the scale of these effects emerge from a bird's 
eye examination of the 3-dimensional figure below. The perspective is such that the viewer is looking from the origin from a point high in the positive orthant, i.e. from a low value for all three axis variables. It is evident that an increase in $\kappa$ has a comparatively smaller impact on the climate policy threshold. Hence, the correct calibration of the noisiness level is more crucial to the decision on the optimal timing of a climate policy than the degree of ambiguity.

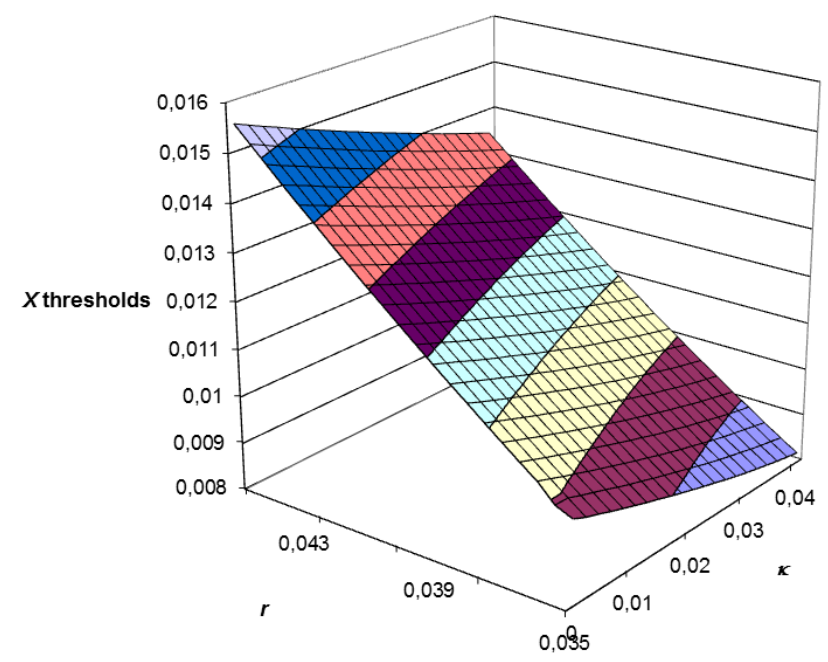

Figure 4: The Climate Policy Thresholds for Simultaneous Changes in the Discount Rate $r$ and $\kappa$

How to calibrate the discount rate is one of the most controversial questions in the economic literature on climate change, e.g. see Stern (2007). As this problem is still far from being resolved, the sensitivity to alternative discounting assumptions needs to be explored. Consider $r$ fixed, Figure 4 shows that Knightian uncertainty again accelerates the climate policy adoption. On the contrary, for a fixed $\kappa$, the simulation confirms the view that higher discount rates bolster the reasons for taking a "wait and see attitude" towards climate policy. This is due to the fact that for a small value of $r$ the particular integral is a good deal bigger and therefore the intertemporal damage is substantially larger. Conversely, a higher discounting factor will trigger a later adoption of climate policy. Figure 4 also reveals that the problematic choice of the discount rate is of more importance to the simulation results than Knightian uncertainty, as the effects caused by a marginal increase of $r$ counteracts the outcome induced by a marginal increase of $\kappa$. This highlights the importance of attaining a consensus on the discount rate before an appraisal on the optimal timing of policy implementation can be achieved.

As already referred to at the beginning of this section, estimations of the abatement costs also face a lot of uncertainties. Figure 5 provides a sensitivity analysis of the thresholds with respect to $w(\tau)$, i.e. we illustrate the impact of alternative climate stabilisation costs upon the threshold. This simulation shows that higher climate stabilisation costs lead to an increase of 


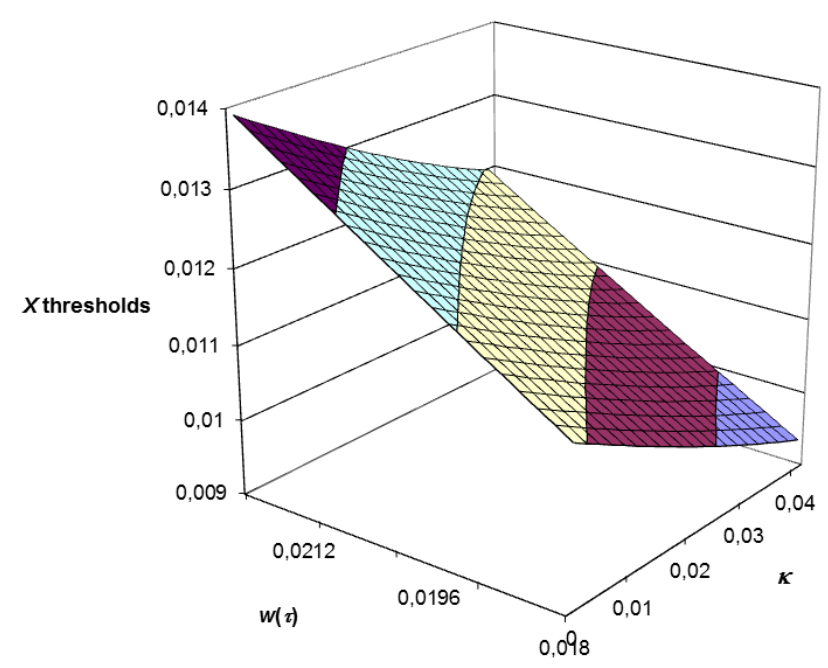

Figure 5: The Climate Policy Thresholds for Alternative Costs of Climate Stabilisation

the no action area, i.e. an increasing $w(\tau)$ increases the climate policy threshold. Intuitively, this makes perfect sense. Higher costs make climate policies less attractive for policy makers, so policy makers hesitate to perform them in the first place. However, the option value of the climate policy opportunity is again lower under Knightian uncertainty than in the standard model. Therefore, an ambiguity-averse policy maker acts earlier. However, changes in the abatement costs have a more influential effect on the timing than changes in Knightian uncertainty.

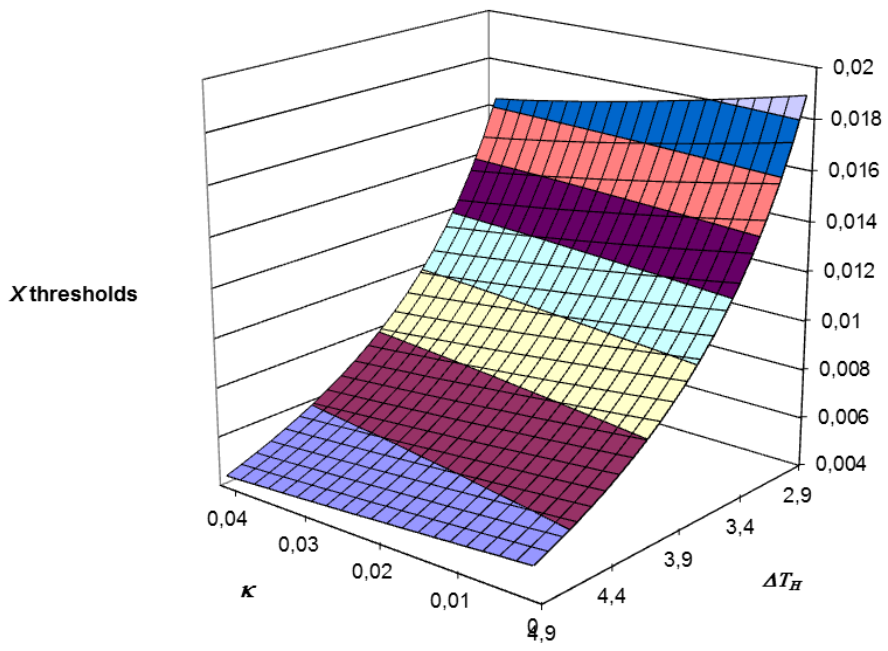

Figure 6: The Impact of Changes in $\Delta \mathbb{T}_{H}$ Upon the Threshold

Finally we analyse how different expected degrees of warming in the business as usual scenario, i.e. changes in $\Delta \mathbb{T}_{H}$, affect the threshold. Looking back on all IPCC assessments, it becomes evident that uncertainty about $\Delta \mathbb{T}_{H}$ could not be resolved significantly in the last years. 
Accounting for different assumptions of $\Delta \mathbb{T}_{H}$, Figure 6 clearly indicates that the tactic to keep options open and await new information about the temperature increase rather than undertake climate policy today becomes less attractive. In other words, higher $\Delta \mathbb{T}_{H}$ values accelerate climate policies by shrinking the no action area. In contrast to the simulations above, an increase of $\Delta \mathbb{T}_{H}$ and $\kappa$ work in the same direction. However, the effects of a change in $\kappa$ are again of secondary importance.

All simulations present similar results. Compared to the assumption of risk, Knightian uncertainty in the damage costs accelerates the climate policy adoption. However, the size of effects caused by Knightian uncertainty is rather small compared to the impacts of variations in the other parameters. Assuming a higher noisiness level, a higher discount rate or higher climate policy costs swamps the effects caused by Knightian uncertainty and thus implies the recommendation to delay the adoption of a climate policy. Likewise, implementing a higher temperature increase in the business as usual scenario exerts a more dominating influence on the policy maker's decision to prevent climate change. Therefore we conclude, that Knightian uncertainty is no "carte blanche" extreme policy activism.

\section{Conclusions}

The modelling of Knightian uncertainty is a relatively unchartered area of climate research. In spite of its clear climate policy relevance, few authors have explored the topic yet. While the paper will be of interest to specialists in real option theory, given the policy importance of the issue in hand we also believe that our assessment of the central question motivating our analysis will be of interest to a wider audience of climate scientists and policy makers. A unifying message from our paper could be stated as follows: We have demonstrated that Knightian uncertainty affects irreversible climate policies in a way which significantly differs from the impact of risk, and that Knightian uncertainty accelerates climate policy. However, our simulations of the model have revealed that Knightian uncertainty does not necessarily imply extreme policy activism. When assuming different but also feasible values for other crucial parameters, the size of effects caused by Knightian uncertainty turns out to be comparatively small. For example, a consensus on the discount rate would have a bigger impact on the policy maker's decision. These insights hold non-trivial value for decision making.

We believe that our application of Knightian uncertainty comes with an advantage and a disadvantage. The advantage is that it allows one to recognise the difference between risk and uncertainty and its implied size of effects. ${ }^{21}$ Thus it provides a more realistic grounding for assessing current climate policy and to derive optimal and rational policy trajectories when fundamental uncertainties and ambiguities are involved. ${ }^{22}$ On the other hand one has

\footnotetext{
${ }^{21}$ To quote from Mastrandrea \& Schneider (2004, p. 571) "we do not recommend that our quantitative results be taken literally, but we suggest that our probabilistic framework and methods be taken seriously". See also Schneider \& Mastrandrea (2005).

${ }^{22}$ Some readers may find the ambiguity and the additional layer of uncertainty psychologically disturbing.
} 
to admit that the comparative static results also have their limitations. First, the numerical results do not account for the fundamental dynamic nature of abatement and mitigation policies. Second, we have focussed on Knightian uncertainty in the damage function. However, there are further layers of uncertainty in complex climate models about which we have ambiguous beliefs. Our analysis may therefore be considered as a first step and it may be refined in several ways. One future research question is the possibility of tipping points. In addition to a high level of complexity, the major challenge of this extension is the need to incorporate thresholds, discontinuities and sudden switches which remain poorly understood on a theoretical level. ${ }^{23}$ Another interesting direction goes towards a more detailed analysis of short- and medium-run climate projections. ${ }^{24}$ We hope to take up some of these tasks in our future work and we consider it probable that this research agenda and the conceptual follow-up issues will continue to warrant substantial research effort in the future.

\section{Acknowledgement}

The research was supported through the Cluster of Excellence "Integrated Climate System Analysis and Prediction" (CliSAP), University of Hamburg, funded through the German Science Foundation (DFG) and the International Max Planck Research School on Earth System Modelling (IMPRS-ESM). The authors are grateful to participants at the CESifo Area Conference on Energy and Climate Economics in Munich (October 2011) and to Hermann Held for helpful comments on an earlier draft.

\section{Appendices}

\section{A Derivation of equation (12)}

First, we to show that the $Q^{\theta} \in \mathscr{P}$ that minimises the expectation value in equation (11) is generated by $\theta=-\kappa$.

We know that $X_{s}(1-\delta) \Delta \mathbb{T}_{s}^{2}$ has a small value so that $\frac{1}{2}\left(X_{s}(1-\delta) \Delta \mathbb{T}_{s}^{2}\right)^{2}$ only adds insignificantly to the term in equation (11). We therefore neglect the quadratic term when minimising the expectation value in the following. ${ }^{25}$

But if the previously agreed modeling framework was wrong and the certainty about appropriate climate policy unjustified, it seems an improvement.

${ }^{23}$ The climate literature on tipping points is, indeed, a fast growing industry. Unfortunately, there are not any models yet incorporating such nonlinearities into micro-founded decision-making frameworks with Knightian uncertainty. It must be emphasised that the model described here is sufficiently general to study various tipping points. It is only necessary to fine-tune the framework for specific nonlinearities and to embed further stochastic processes.

${ }^{24}$ In Figure $2-6$ the impact of Knightian uncertainty is "statically" addressed. Hence, we may next aim to study the temporal implications of Knightian uncertainty, and the impact of less medium-run ambiguity resulting from more reliable decadal projections upon optimal climate policies.

${ }^{25}$ We only show the proof for the 1st order term for the "whole exponential loss function." Small $X$ means that taylor's expansions are a good approximation. As the policymaker takes a pessimistic view of the loss function, it is intuitive and straightforward that the lowest value of $\kappa$ for the loss function shall be obtained. 
Additionally Fubini's theorem for conditional expectations transforms $W^{\mathrm{N}}\left(X, \Delta \mathbb{T} ; \Delta \mathbb{T}_{H}\right)$ to

$$
\frac{1}{1-\delta} \min _{Q^{\theta} \in \mathscr{P}} \int_{t=0}^{\infty} e^{-\left(r-(1-\delta) g_{0}\right) s} E^{Q^{\theta}}\left[1-X_{s}(1-\delta) \Delta \mathbb{T}_{s}^{2} \mid \mathscr{F}_{t}\right] d s
$$

By applying Itô's Lemma to the logarithm of $X_{s}$ we obtain $\forall s \geq 0$ :

$$
X_{s}=X_{0} e^{\left(\alpha-\frac{1}{2} \sigma^{2}-\sigma \theta\right) s+\sigma B_{s}^{\theta}}=X_{0} e^{\left(\alpha-\frac{1}{2} \sigma^{2}-\sigma \theta\right) s} e^{\sigma B_{s}^{\theta}} .
$$

Obviously it holds that

$$
X_{s}=X_{0} e^{\left(\alpha-\frac{1}{2} \sigma^{2}-\sigma \theta\right) s} e^{\sigma B_{s}^{\theta}} \leq X_{0} e^{\left(\alpha-\frac{1}{2} \sigma^{2}+\sigma \kappa\right) s} e^{\sigma B_{s}^{\theta}} \quad \forall s \geq 0, \quad \forall \theta \in[-\kappa, \kappa] .
$$

Due to the monotonicity of the conditional expectation value, we obtain

$$
\begin{aligned}
& E^{Q^{\theta}}\left[1-X_{0} e^{\left(\alpha-\frac{1}{2} \sigma^{2}-\sigma \theta\right) s} e^{\sigma B_{s}^{\theta}}(1-\delta) \Delta \mathbb{T}_{s}^{2} \mid \mathscr{F}_{t}\right] \\
& \geq E^{Q^{\theta}}\left[1-X_{0} e^{\left(\alpha-\frac{1}{2} \sigma^{2}+\sigma \kappa\right) s} e^{\sigma B_{s}^{\theta}}(1-\delta) \Delta \mathbb{T}_{s}^{2} \mid \mathscr{F}_{t}\right] \\
& =\left(1-X_{0} e^{\left(\alpha-\frac{1}{2} \sigma^{2}+\sigma \kappa\right) s}\right)(1-\delta) \Delta \mathbb{T}_{s}^{2} E^{Q^{\theta}}\left[e^{\sigma B_{s}^{\theta}} \mid \mathscr{F} t\right] \\
& =\left(1-X_{0} e^{\left(\alpha-\frac{1}{2} \sigma^{2}+\sigma \kappa\right) s}\right)(1-\delta) \Delta \mathbb{T}_{s}^{2} e^{\frac{1}{2} \sigma^{2} s} \\
(\text { A. } 4) & =\left(1-X_{0} e^{\left(\alpha-\frac{1}{2} \sigma^{2}+\sigma \kappa\right) s}\right)(1-\delta) \Delta \mathbb{T}_{s}^{2} E^{Q^{-\kappa}}\left[e^{\sigma B_{s}^{-\kappa}} \mid \mathscr{F} t\right] \quad \forall \mathrm{s} \geq 0, \forall \theta \in[-\kappa, \kappa] .
\end{aligned}
$$

Thus, the measure $Q^{-\kappa} \in \mathscr{P}$ minimises the expectation value in (11), which we therefore denote as $Q^{*}$. Consequently the process $X$ that results from implementing $\theta=-\kappa$ into equation (8) shall be called $X^{*}$.

For the following considerations let $W^{\mathrm{N}}\left(X, \Delta \mathbb{T} ; \Delta \mathbb{T}_{H}\right)$ be conveniently abbreviated by $W^{\mathrm{N}}$. The corresponding Hamilton-Jacobi-Bellman equation to equation (11) is as follows (see for example chapter 3.1. in Stokey (2009) as an introduction to the Hamilton-Jacobi-Bellman equation):

$$
\begin{aligned}
\left(r-(1-\delta) g_{0}\right) W^{\mathrm{N}} & =\frac{1}{1-\delta}\left(1-X^{*}(1-\delta) \Delta \mathbb{T}^{2}+\frac{1}{2}\left(X^{*}(1-\delta) \Delta \mathbb{T}^{2}\right)^{2}\right) \\
& +\frac{1}{d t} E^{Q^{*}}\left[d W^{N} \mid \mathscr{F}_{t}\right] \\
& =\frac{1}{1-\delta}-X^{*} \Delta \mathbb{T}^{2}+\frac{1}{2} X^{*^{2}}(1-\delta) \Delta \mathbb{T}^{4}+\frac{1}{d t} E^{Q^{*}}\left[d W^{N} \mid \mathscr{F}_{t}\right]
\end{aligned}
$$

$W^{\mathrm{N}}$ is obviously differentiable at least once in $\Delta \mathbb{T}$ and twice in $X^{*}$, which allows to apply

However, the exponential form of the loss function prevents us from obtaining the proof. We therefore opt to focus attention upon the first order term to show the choice of the lowest value of kappa by the policy maker, while ignoring the comparably smaller 2nd-order term, without the loss of economic intuition. 
Itô's Lemma:

$$
\begin{aligned}
d W^{\mathrm{N}}= & \frac{\partial W^{\mathrm{N}}}{\partial \Delta \mathbb{T}} d \Delta \mathbb{T}+\frac{\partial W^{\mathrm{N}}}{\partial X^{*}} d X^{*}+\frac{\partial^{2} W^{\mathrm{N}}}{\partial X^{*^{2}}}\left(d X^{*}\right)^{2} \\
= & \frac{\ln (2)}{H}\left(2 \Delta \mathbb{T}_{H}-\Delta \mathbb{T}_{t}\right) \frac{\partial W^{\mathrm{N}}}{\partial \Delta \mathbb{T}} d t+\frac{\partial W^{\mathrm{N}}}{\partial X^{*}}\left[(\alpha+\sigma \kappa) X_{t}^{*} d t+\sigma X_{t}^{*} d B_{t}^{-\kappa}\right] \\
& +\frac{1}{2} \sigma^{2} X^{*^{2}} \frac{\partial^{2} W^{\mathrm{N}}}{\partial X^{*^{2}}} d t,
\end{aligned}
$$

by using equation (4) in the text. Taking expectation of (A6) and dividing by $d t$ we obtain

$$
\frac{E\left[d W^{\mathrm{N}}\right]}{d t}=\frac{\ln (2)}{H}\left(2 \Delta \mathbb{T}_{H}-\Delta \mathbb{T}_{t}\right) \frac{\partial W^{\mathrm{N}}}{\partial \Delta \mathbb{T}}+(\alpha+\kappa \sigma) X_{t}^{*} \frac{\partial W^{\mathrm{N}}}{\partial X^{*}}+\frac{1}{2} \sigma^{2} X^{*^{2}} \frac{\partial^{2} W^{\mathrm{N}}}{\partial X^{*^{2}}} .
$$

Substituting (A7) back to the Hamilton-Jacobi-Bellman equation (A5) gives

$$
\left(r-(1-\delta) g_{0}\right) W^{\mathrm{N}}=\frac{1}{1-\delta}-X^{*} \Delta \mathbb{T}^{2}+\frac{1}{2} X^{*^{2}}(1-\delta) \Delta \mathbb{T}^{4}
$$

$$
+\frac{\ln (2)}{H}\left(2 \Delta \mathbb{T}_{H}-\Delta \mathbb{T}\right) \frac{\partial W^{\mathrm{N}}}{\partial \Delta \mathbb{T}}+(\alpha+\kappa \sigma) X^{*} \frac{\partial W^{\mathrm{N}}}{\partial X^{*}}+\frac{1}{2} \sigma^{2} X^{2} \frac{\partial^{2} W^{\mathrm{N}}}{\partial X^{*^{2}}},
$$

which is equation (12) in the text.

\section{B Particular solutions to $W^{\mathrm{NP}}$ and $W^{\mathrm{A}}$}

Using equations (11) and (5) yields the following particular integral,

$W^{\mathrm{NP}}\left(X, \Delta \mathbb{T} ; \Delta \mathbb{T}_{H}\right)=\frac{1}{1-\delta} \times$

$\int_{t=0}^{\infty}\left[1-\sum_{i=1}^{2} \frac{(-1)^{i+1}}{i !} X^{*^{i}} e^{\left[i(\alpha+\kappa \sigma)+\frac{1}{2} i(i-1) \sigma^{2}\right] s}(1-\delta)^{i}\left(2 \Delta \mathbb{T}_{H}\left(1-e^{-\frac{\ln 2}{H} s}\right)\right)^{2 i}\right] e^{-\left(r-(1-\delta) g_{0}\right) s} d s$.

In the same manner we employ equation (16) and (20) to derive

$W^{\mathrm{A}}(X, \Delta \mathbb{T} ; \tau)=\frac{(1-w(\tau))^{1-\delta}}{1-\delta} \times$

$\int_{t=0}^{\infty}\left[1-\sum_{i=1}^{2} \frac{(-1)^{i+1}}{i !} X^{*^{i}} e^{\left[i(\alpha+\kappa \sigma)+\frac{1}{2} i(i-1) \sigma^{2}\right] s}(1-\delta)^{i}\left(2 \Delta \mathbb{T}_{H}\left(1-e^{-\frac{\ln 2}{H} s}\right)\right)^{2 i}\right] e^{-\left(r-(1-\delta) g_{0}\right) s} d s$.

Equations (B1) and (B2) result from Itô's Lemma which means that equation (A2) with $\theta=-\kappa$ is applied to equation (11) and (16), respectively. Furthermore please note that 
$E^{Q^{-\kappa}}\left[e^{\sigma B_{s}^{-\kappa}} \mid \mathscr{F}_{t}\right]=e^{\frac{1}{2} \sigma^{2} s}$. By expanding the terms

$$
\left(1-e^{-\frac{\ln 2}{H} t}\right)^{2}=1-2 e^{-\frac{\ln 2}{H} t}+e^{-2 \frac{\ln 2}{H} t}
$$

and

$$
\left(1-e^{-\frac{\ln 2}{H} t}\right)^{4}=1-4 e^{-\frac{\ln 2}{H} t}+6 e^{-2 \frac{\ln 2}{H} t}-4 e^{-3 \frac{\ln 2}{H} t}+e^{-4 \frac{\ln 2}{H} t},
$$

we obtain

$$
\begin{aligned}
& {\left[1-\sum_{i=1}^{2} \frac{(-1)^{i+1}}{i !} X^{*^{i}} e^{\left[i(\alpha+\kappa \sigma)+\frac{1}{2} i(i-1) \sigma^{2}\right] s}(1-\delta)^{i}\left(2 \Delta \mathbb{T}_{H}\left(1-e^{-\frac{\ln 2}{H} s}\right)\right)^{2 i}\right] e^{-\left(r-(1-\delta) g_{0}\right) s}} \\
& =e^{-\left(r-(1-\delta) g_{0}\right) s}-4 \Delta \mathbb{T}_{H}^{2}(1-\delta) X^{*} e^{(\alpha+\kappa \sigma) s}\left(1-2 e^{-\frac{\ln 2}{H} s}+e^{-2 \frac{\ln 2}{H} s}\right) e^{-\left(r-(1-\delta) g_{0}\right) s} \\
& +8 \Delta \mathbb{T}_{T}^{4}(1-\delta)^{2} X^{*^{2}} e^{\left[2(\alpha+\kappa \sigma)+\sigma^{2}\right] s} \times \\
& \left(1-4 e^{-\frac{\ln 2}{H} s}+6 e^{-2 \frac{\ln 2}{H} s}-4 e^{-3 \frac{\ln 2}{H} s}+e^{-4 \frac{\ln 2}{H} s}\right)^{4} e^{-\left(r-(1-\delta) g_{0}\right) s} .
\end{aligned}
$$

Substituting (B5) back into (B1) and integrating yields

$$
\begin{aligned}
& W^{\mathrm{NP}}\left(X, \Delta \mathbb{T} ; \Delta \mathbb{T}_{H}\right)=\frac{1}{1-\delta}\left[\frac{1}{r-(1-\delta) g_{0}}-4 \Delta \mathbb{T}_{H}^{2}(1-\delta) X^{*}\left(\frac{1}{\eta_{1}}-\frac{2}{\eta_{1}+\frac{\ln 2}{H}}+\frac{1}{\eta_{1}+2 \frac{\ln 2}{H}}\right)\right. \\
& \begin{array}{l}
\text { (B.6) } \left.\quad+8 \Delta \mathbb{T}_{H}^{4}(1-\delta)^{2} X^{*^{2}}\left(\frac{1}{\eta_{2}}-\frac{4}{\eta_{2}+\frac{\ln 2}{H}}+\frac{6}{\eta_{2}+2 \frac{\ln 2}{H}}-\frac{4}{\eta_{2}+3 \frac{\ln 2}{H}}+\frac{1}{\eta_{2}+4 \frac{\ln 2}{H}}\right)\right]
\end{array}
\end{aligned}
$$

where

$$
\begin{aligned}
& \eta_{1}=r-(1-\delta) g_{0}-(\alpha+\kappa \sigma), \\
& \eta_{2}=r-(1-\delta) g_{0}-\left(2(\alpha+\kappa \sigma)+\sigma^{2}\right) .
\end{aligned}
$$

Similarly, we have

$$
\begin{aligned}
& W^{\mathrm{A}}(X, \Delta \mathbb{T} ; \tau)=\frac{(1-w(\tau))^{1-\delta}}{1-\delta}\left[\frac{1}{r-(1-\delta) g_{0}}-4 \Delta \tau^{2}(1-\delta) X^{*}\left(\frac{1}{\eta_{1}}-\frac{2}{\eta_{1}+\frac{\ln 2}{H}}+\frac{1}{\eta_{1}+2 \frac{\ln 2}{H}}\right)\right. \\
& \left.+8 \Delta \tau^{4}(1-\delta)^{2} X^{*^{2}}\left(\frac{1}{\eta_{2}}-\frac{4}{\eta_{2}+\frac{\ln 2}{H}}+\frac{6}{\eta_{2}+2 \frac{\ln 2}{H}}-\frac{4}{\eta_{2}+3 \frac{\ln 2}{H}}+\frac{1}{\eta_{2}+4 \frac{\ln 2}{H}}\right)\right],
\end{aligned}
$$

which are equations (21) and (22) in the text, respectively. 


\section{General Solution $W^{\mathrm{NG}}$ for $W^{\mathrm{N}}$}

We guess the solution to equation (14) has the following functional form:

$$
W^{\mathrm{NG}}\left(t=0, X, \Delta \mathbb{T} ; \Delta \mathbb{T}_{H}\right)=A X^{*^{\beta}}\left(\Delta \mathbb{T}^{2}+C \Delta \mathbb{T}+D\right)
$$

where $A, C, \mathrm{D}$ are some parameters. Calculating derivatives, we obtain

$$
\begin{aligned}
& \frac{\partial W^{\mathrm{NG}}}{\partial \Delta \mathbb{T}}=A X^{*^{\beta}}(2 \Delta \mathbb{T}+C) \\
& X^{*} \frac{\partial W^{\mathrm{NG}}}{\partial X^{*}}=\beta A X^{*^{\beta}}\left(\Delta \mathbb{T}^{2}+C \Delta \mathbb{T}+D\right) \quad \text { and } \\
& X^{*^{2}} \frac{\partial^{2} W^{\mathrm{NG}}}{\partial X^{*^{2}}}=\beta(\beta-1) A X^{*^{\beta}}\left(\Delta \mathbb{T}^{2}+C \Delta \mathbb{T}+D\right) .
\end{aligned}
$$

Substituting equations $(\mathrm{C} 1)$ - (C4) back to equation (14) and rearranging yields

$$
\begin{aligned}
& 2\left(\frac{\ln (2)}{H}\right) A X^{*^{\beta}}\left(\Delta \mathbb{T}^{2}-\left(2 \Delta \mathbb{T}_{H}-\frac{C}{2}\right) \Delta \mathbb{T}-C \Delta \mathbb{T}_{H}\right) \\
& =\left[-\left(r-(1-\delta) g_{0}\right)+(\alpha+\kappa \sigma) \beta+\frac{1}{2} \sigma^{2} \beta(\beta-1)\right] A X^{*^{\beta}}\left(\Delta \mathbb{T}^{2}+C \Delta \mathbb{T}+D\right) .
\end{aligned}
$$

Solving (C5) requires $\Delta \mathbb{T}^{2}-\left(2 \Delta \mathbb{T}_{H}-\frac{C}{2}\right) \Delta \mathbb{T}-C \Delta \mathbb{T}_{H}=\left(\Delta \mathbb{T}^{2}+C \Delta \mathbb{T}+D\right)$. Thus, we have

$$
C=-4 \Delta \mathbb{T}_{H}
$$

and

$$
D=-C \Delta \mathbb{T}_{H}=4 \Delta \mathbb{T}_{H}^{2}
$$

Plugging (C6) and (C7) into (C5), we obtain

$$
\left[-\left(r-(1-\delta) g_{0}+2\left(\frac{\ln (2)}{H}\right)\right)+(\alpha+\kappa \sigma) \beta+\frac{1}{2} \sigma^{2} \beta(\beta-1)\right] W^{\mathrm{NG}}=0,
$$

where $W^{\mathrm{NG}}=A X^{*^{\beta}}\left(\Delta \mathbb{T}^{2}-4 \Delta \mathbb{T}_{H} \Delta \mathbb{T}+4 \Delta \mathbb{T}_{H}^{2}\right)$. The solution of (C8) requires

$$
(\alpha+\kappa \sigma) \beta+\frac{1}{2} \sigma^{2} \beta(\beta-1)-\left(r-(1-\delta) g_{0}+2\left(\frac{\ln (2)}{H}\right)\right)=0
$$

Let $\beta_{1}$ and $\beta_{1}$ be the positive and negative roots of the above characteristic function, respectively. By some manipulations, this leads to

$$
W^{\mathrm{NG}}=A_{1} X^{*^{\beta_{1}}}\left(\Delta \mathbb{T}^{2}-4 \Delta \mathbb{T}_{H} \Delta \mathbb{T}+4 \Delta \mathbb{T}_{H}^{2}\right)-A_{2} X^{*^{\beta_{2}}}\left(\Delta \mathbb{T}^{2}-4 \Delta \mathbb{T}_{H} \Delta \mathbb{T}+4 \Delta \mathbb{T}_{H}^{2}\right)
$$


As we only consider the option to take action, we need to set the boundary condition such that $\lim _{X \rightarrow 0} W^{\mathrm{NG}}(X)=0$, which is tantamount to a zero option value of a climate policy, if climate change causes no damages that reduce the GDP. Therefore, the general solution with the negative root can be ignored. Consequently, we obtain

$$
W^{\mathrm{NG}}=A_{1} X^{*^{\beta_{1}}}\left(\Delta \mathbb{T}^{2}-4 \Delta \mathbb{T}_{H} \Delta \mathbb{T}+4 \Delta \mathbb{T}_{H}^{2}\right)
$$

\section{References}

Asano, T. (2010). Precautionary principle and the optimal timing of environmental policy under ambiguity. Environmental and Resource Economics, 47, 173-196.

Becker, S. W., \& Brownson, F. O. (1964). What price ambiguity? Or the role of ambiguity in decision-making. Journal of Political Economy, 72, pp. 62-73.

Bertola, G. (2010). Options, inaction, and uncertainty. Scottish Journal of Political Economy, $57,254-271$.

Borsuk, M., \& Tomassini, L. (2005). Uncertainty, imprecision, and the precautionary principle in climate change assessment. Water Science \&5 Technology, 52, 213-225.

Camerer, C., \& Weber, M. (1992). Recent developments in modeling preferences: Uncertainty and ambiguity. Journal of Risk and Uncertainty, 5, 325-370.

Chen, Z., \& Epstein, L. (2002). Ambiguity, risk, and asset returns in continuous time. Econometrica, 70, pp. 1403-1443.

Dixit, A. K., \& Pindyck, R. S. (1994). Investment under Uncertainty. Princeton University Press.

Edenhofer, O., Knopf, B., Barker, T., Baumstark, L., , Bellevrat, E., Chateau, B., Criqui, P., Isaac, M., Kitous, A., Kypreos, S., Leimbach, M., Lessmann, K., Magné, B., Scrieciu, S., Turton, H., \& van Vuuren, D. P. (2010). The economics of low stabilization: Model comparison of mitigation strategies and costs. The Energy Journal, 31, 11-48.

Ellsberg, D. (1961). Risk, ambiguity, and the Savage axioms. The Quarterly Journal of Economics, 75, 643-669.

Funke, M., \& Paetz, M. (2011). Environmental policy under model uncertainty: a robust optimal control approach. Climatic Change, 107, 225-239.

Gilboa, I., \& Schmeidler, D. (1989). Maxmin expected utility with non-unique prior. Journal of Mathematical Economics, 18, 141 - 153.

Halevy, Y. (2007). Ellsberg revisited: An experimental study. Econometrica, 75, 503-536. 
Hogarth, R. M., \& Kunreuther, H. (1985). Ambiguity and insurance decisions. The American Economic Review, 75, pp. 386-390.

Hsu, M., Bhatt, M., Adolphs, R., Tranel, D., \& Camerer, C. F. (2005). Neural systems responding to degrees of uncertainty in human decision-making. Science, 310, 1680-1683.

Kriegler, E., \& Held, H. (2005). Utilizing belief functions for the estimation of future climate change. International Journal of Approximate Reasoning, 39, 185 - 209.

Leahy, J. V. (1993). Investment in competitive equilibrium: The optimality of myopic behavior. The Quarterly Journal of Economics, 108, pp. 1105-1133.

Mastrandrea, M. D., \& Schneider, S. H. (2004). Probabilistic integrated assessment of "dangerous" climate change. Science, 304, 571-575.

Mendelsohn, R., Schlesinger, M., \& Williams, L. (2000). Comparing impacts across climate models. Integrated Assessment, 1, 37-48.

Millner, A., Dietz, S., \& Heal, G. (2010). Ambiguity and Climate Policy. Working Paper 16050 National Bureau of Economic Research.

Nishimura, K. G., \& Ozaki, H. (2007). Irreversible investment and Knightian uncertainty. Journal of Economic Theory, 136, 668 - 694.

Nordhaus, W. D., \& Boyer, J. (2000). Warming the World: Economic Models of Global Warming. (Cambridge, Massachusetts: MIT Press).

Pindyck, R. S. (2009a). Modeling the impact of warming in climate change economics. In The Economics of Climate Change: Adaptations Past and Present (pp. 47-71). University of Chicago Press.

Pindyck, R. S. (2009b). Uncertain Outcomes and Climate Change Policy. Working Paper 15259 National Bureau of Economic Research.

Reichert, P. (1997). On the necessity of using imprecise probabilities for modelling environental systems. Water Science \& Technology, 36, 149 - 156.

Roe, G. H., \& Baker, M. B. (2007). Why is climate sensitivity so unpredictable? Science, $318,629-632$.

Sarin, R. K., \& Weber, M. (1993). Effects of ambiguity in market experiments. Management Science, 39, pp. 602-615.

Savage, L. (1954). The Foundations of Statistics. New York: Wiley. 
Schneider, S. H., \& Mastrandrea, M. D. (2005). Probabilistic assessment of "dangerous" climate change and emissions pathways. Proceedings of the National Academy of Sciences of the United States of America, 102, 15728-15735.

Smith, V. L. (1969). Measuring nonmonetary utilities in uncertain choices: The Ellsberg urn. The Quarterly Journal of Economics, 83, pp. 324-329.

Stern, N. (2007). The Economics of Climate Change: The Stern Review. Cambridge University Press.

Stokey, N. L. (2009). The Economics of Inaction: Stochastic Control Models with Fixed Costs. Princeton University Press.

Tol, R. S. (2002). Estimates of the damage costs of climate change. part 1: Benchmark estimates. Environmental and Resource Economics, 21, 47-73.

Trojanowska, M., \& Kort, P. (2010). The worst case for real options. Journal of Optimization Theory and Applications, 146, 709-734.

Vardas, G., \& Xepapadeas, A. (2010). Model uncertainty, ambiguity and the precautionary principle: Implications for biodiversity management. Environmental and Resource Economics, 45, 379-404.

Weitzman, M. L. (2009a). Additive damages, fat-tailed climate dynamics, and uncertain discounting. Economics: The Open-Access, Open-Assessment E-Journal, 3, 2009-39.

Weitzman, M. L. (2009b). On modeling and interpreting the economics of catastrophic climate change. The Review of Economics and Statistics, 91, 1-19. 